\title{
Photodynamic diagnosis - current tool in diagnosis of carcinoma in situ of the urinary bladder
}

\author{
Marek Ireneusz Lipiński, Waldemar Różański, Michał Paweł Markowski
}

$2^{\text {nd }}$ Clinic of Urology Medical University of Łódź

Contemp Oncol (Pozn) 2015; 19 (4): 341-342

DOI: $10.5114 /$ wo.2015.54391

Carcinoma in situ (CIS) of the urinary bladder is extremely hard to diagnose. The symptoms are highly unspecific and the small, flat CIS lesions can easily be missed, thus remaining unseen in standard white light cystoscopy. Photodynamic diagnosis (PDD) is recommended by the European Association of Urology (EAU) as a diagnostic procedure in cases of suspected CIS [1].

A 58-year-old man visited the outpatient clinic complaining of symptoms of dysuria and occasional pain during micturition. The patient had been a smoker for 20 years, with no risk of exposure to chemical substances. The medical history did not indicate any haematuria or fever. Digital rectal examination found the prostate to be of proper density, with little enlargement and no other pathological findings. The preliminary diagnosis was set as lower urinary tract syndrome secondary to prostate enlargement. The patient was sent for creatinine assay, prostate specific antigen (PSA) assay, a urine test, and ultrasound examination of the kidneys, bladder, and prostate. He received a prescription for diclofenac, tamsulosin, and ciprofloxacin.

The next appointment in the outpatient clinic was set for two months later as a follow-up. During that visit, the patient reported two episodes of painless bleeding in his urine. The serum levels of creatinine and PSA were 0.9 and $1.2 \mathrm{ng} / \mathrm{ml}$, respectively, and no abnormalities were observed in the results of the urine test. No pathological changes were observed by ultrasonography. After the treatment, the symptoms of dysuria were diminished.

In response to the presence of bleeding in the urine, the patient was sent for urethrocystoscopy, performed with short intravenous anaesthesia, and urinary cytology, which revealed the presence of pathological cells. On the basis of this result and that of the earlier ultrasonography, which indicated no bladder tumour symptoms, the patient was qualified for PDD.

Photodynamic diagnosis was performed after intravesical instillation of a photosensitiser (Hexvix $\left.{ }^{\circledR}\right) 60$ minutes before the procedure. A Wolf PDD system was used to observe the photodynamic effect. Although the white light cystoscopy revealed only one pathological lesion, $4 \mathrm{~mm}$ in diameter, on the front wall of the urinary bladder, the uri- nary bladder mucosa seemed otherwise unaffected by any pathological changes. After switching to PDD mode, three independent points were indicated by red flashes: one on the back wall (8 $\mathrm{mm}$ in diameter), one on the right wall (5 $\mathrm{mm}$ in diameter), and one on the left wall of the urinary bladder (5 $\mathrm{mm}$ in diameter) (photo). Tissue samples were taken from each of the suspicious lesions. Both the papillary tumour and the three lesions visible only in PDD were treated by transurethral resection of the bladder tumour (TURBT). After the operation, instillation of mitomycin C was performed.

The histopathological report revealed the papillary tumour from the front wall to be a high-grade non-muscle-invasive bladder cancer (G2) without lamina propria infiltration, the lesions on the back and left walls were found to be carcinoma in situ, and the one on the right wall was a high-grade dysplasia. The patient was immediately qualified to immunotherapy with BCG.

In common practice, the diagnosis of urinary bladder $\mathrm{CIS}$ with non-specific symptoms often presents a problem [2]. Although urinary cytology has high sensitivity for CIS in cases such as specific and non-specific inflammatory processes in the urinary bladder, it can give false positive results. In addition, the result is highly dependent on the experience of the pathologist $[3,4]$. Although CIS can appear to be a flat lesion in white light cystoscopy, with oedema of the urothelium and with abnormal blood vessel patterns, there is, generally speaking, no single standard picture of CIS. Hence, there is a strong need for diagnostic tools that can be used to visualise CIS when it is presented in the urinary bladder.

Photodynamic diagnosis is a tool that can be used in the diagnosis of CIS as part of the TURBT (Transurethral Reaction of Bladder Tumour) procedure and re-TURBT, particularly in combination with Hexvix [5]. In a multicentre study on 286 patients, PDD examination identified CIS lesions in 80 patients (96\%), and white light cystoscopy allowed for identification of CIS in 64 patients (80\%) [6]. Another multicentre study conducted in the USA also compared standard white light cystoscopy and PDD in a group of 311 patients. Statistical analysis was performed on 196 patients. CIS was diagnosed in 56 cases. Solitary lesions 

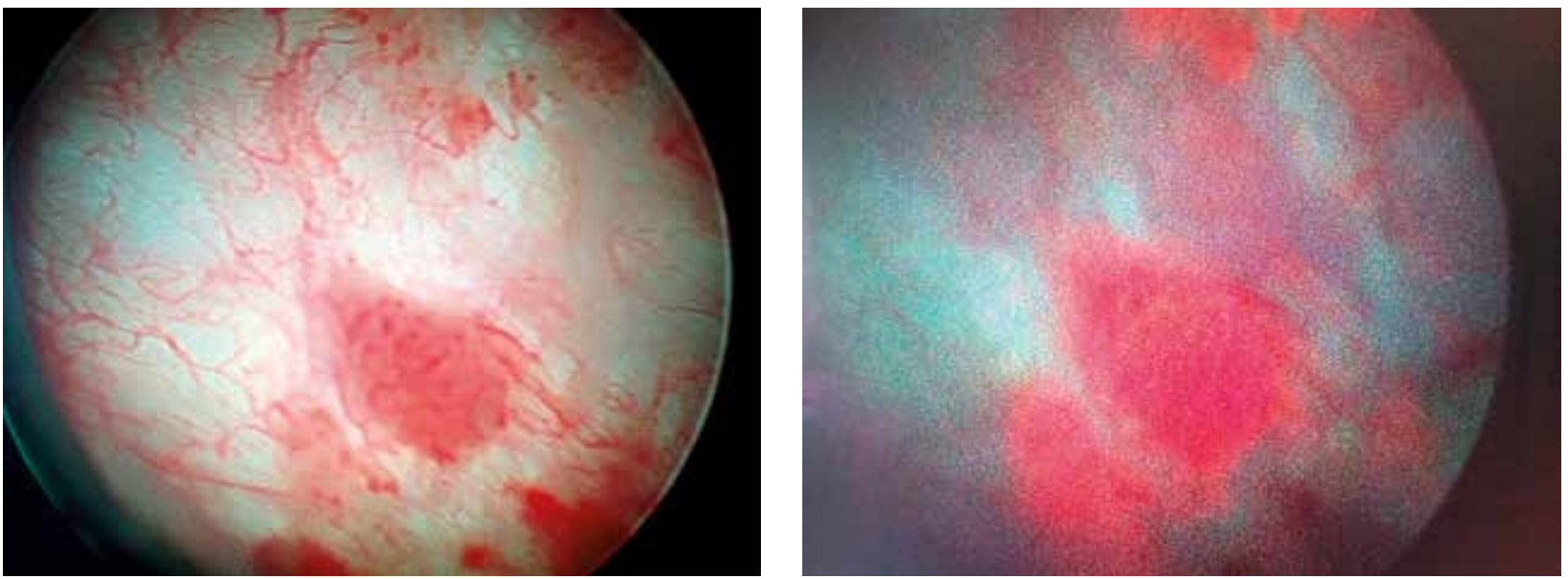

Fig. 1. Image of Cis lesion in white light cystoscopy Image of Cis lesion in PDD mode

were identified in 18 cases, and carcinoma in situ coexisting with papillary tumours in 36 cases. While PDD identified $92 \%$ of the CIS cases, white light cystoscopy identified $68 \%[7,8]$.

A disadvantage of PDD is its rather low specificity, ranging from $35 \%$ to $66 \%$, which is often due to a lack of experience on the part of the operator or the presence of scars after previous TURBT or intravesical instillation [9]. Nevertheless, PDD is recommended as a diagnostic tool in follow-up examinations of patients with CIS after BCG immunotherapy [10]. In a study of 49 patients with CIS examined after BCG therapy, 18 cases of recurrence were noted. Of these 18 cases, white light cystoscopy revealed no CIS lesions, while PDD diagnosed 14. Overall, while nine of the PDD results were found to be false positives (33.3\%), only one positively identified by white light cystoscopy was false (7.1\%) [10].

PDD represents a great enhancement of the urological diagnosis of CIS of the urinary bladder and is a superior method to standard white light cystoscopy in cases where $\mathrm{CIS}$ is suspected. This efficiency is even more important when considering that a diagnosis of CIS demands the quick implementation of appropriate therapy.

This article was supported by scientific grant IPSEN (Warsaw, Poland).

\section{References}

1. Babjuk M, Böhle A, Burger M, et al. Guidelines on Non-muscle invasive bladder cancer (Tar, T1 and Cis). European Association of Urology Guidelines 2015; 13: 913. Available at: http://www. abnoba.de/fileadmin/Upload/Germany/DocCheck/Guideline_ Non-muscle-Invasive-BC_TaT1_LR1.pdf.

2. Kurth KH, Schellhammer PF, OKajima E, et al. Current methods of assessing and treating carcinoma in situ of the bladder with or without involvement of the prostatic urethra. Int J Urol 1995; 2 (Suppl. 2): 8-22.

3. Lokeshwar VB, Habuchi T, Grossman HB, et al. Bladder tumor markers beyond cytology: International Consensus Panel on bladder tumor markers. Urology 2005; 66 (6 Suppl 1): 35-63.

4. Raab Ss, Grzybicki DM, Vrbin CM, Geisinger KR. Urine cytology discrepancies: frequency, causes, and outcomes. Am J Clin Pathol 2007; 127: 946-53.
5. Kriegmair M, Baumgartner R, Knuechel R, Steinbach P, Ehsan A, Lumper W, Hofstädter F, Hofstetter A.. Fluorescence photodetection of neoplastic urothelial lesions following intravesical instillation of 5-aminolevulinic acid. Urology 1994; 44: 836-41.

6. Schmidbauer J, Witjes F, Schmeller N, Donat R, Susani M, Marberger M; Hexvix PCB301/01 Study Group. Improved detection of urothelial carcinoma in situ with hexaminolevulinate fluorescence cystoscopy. J Urol 2004; 171: 135-8.

7. Grossman HB, Gomella L, Fradet Y, et al. PC B302/01 Study Group. A phase III, multicenter comparison of hexaminolevulinate fluorescence cystoscopyand white light cystoscopy for the detection of superficial papillary lesions inpatients with bladder cancer. J Urol 2007; 178: 62-7.

8. Fradet Y, Grossman HB, Gomella L, Lerner S, Cookson M, Albala D, Droller MJ; PC B302/01 Study Group. A comparison of hexaminolevulinate fluorescence cystoscopy and white light cystoscopy for the detection of carcinoma in situ in patients with bladder cancer: a phase III, multicenter study. J Urol 2007; 178: 68-73.

9. Jocham D, Stepp H, Waidelich R. Photodynamic diagnosis in urology: state-of-the-art. Eur Urol 2008; 53: 1138-48.

10. Colombo R, Naspro R, Bellinzoni P, Fabbri F, Guazzoni G, Scattoni V, Losa A, Rigatti P. Photodynamic diagnosis for follow-up of carcinoma in situ of the bladder. Ther Clin Risk Manag 2007; 3: 1003-7.

\section{Address for correspondence}

\section{Marek Lipiński}

$2^{\text {nd }}$ Clinic of Urology Medical University of Łódź

Pabianicka 62

93-513 Lodz, Poland

e-mail: miklipa@poczta.onet.pl 